\title{
Desenvolvimento motor em escolares praticantes e não praticantes de dança*
}

Daniela Bastos de Almeida Rodrigues**

Ignácio Antônio Seixas-da-Silva ${ }^{* * *}$

Rosana Dias de Oliveira Brum ${ }^{* * * *}$

Ricardo Mariano Dublasievicz ${ }^{* * * * *}$

Vitor Ayres Principe $e^{* * * * * * *}$

Sérgio Medeiros Pinto ${ }^{* * * * * * *}$

Rodolfo de Alkmim Moreira Nunes ${ }^{* * * * * * * * *}$

Carlos Soares Pernambuco ${ }^{* * * * * * * * * * *}$

Rodrigo Gomes de Souza do Vale ${ }^{* * * * * * * * * * *}$

Recibido :02 de septiembre de 2020

Aceptado: 15 de mayo de 2021

\footnotetext{
* Artigo original. Sem financiamento. Universidade Estácio de Sá, Cabo Frio, Rio de Janeiro, Brasil.

** Bacharela, professora, Universidade Estácio de Sá, Cabo Frio, Rio de Janeiro, Brasil. Correio eletrônico: danielaalmeidaef@outlook.com; ORCID: https://orcid.org/0000-0001-7409-5370

*** Doutor, Universidade Estácio de Sá, Cabo Frio, Rio de Janeiro, Brasil. Correio eletrônico: ignacio.silva@estacio.br; ORCID https://orcid.org/0000-0002-8151-3910

**** Mestra, Universidade Estácio de Sá, Cabo Frio, Rio de Janeiro, Brasil. Correio eletrônico: rosanahidro@hotmail.com; ORCID https://orcid.org/0000-0001-6147-5673

***** Mestre, Universidade Estácio de Sá, Cabo Frio, Rio de Janeiro, Brasil. Correio eletrônico: ricardo.dublasievicz@estacio.br; ORCID https://orcid.org/0000-0002-0639-7278

${ }^{* * * * * *}$ Mestre, Universidade Estácio de Sá, Cabo Frio, Rio de Janeiro, Brasil. Correio eletrônico: vitor.principe@estacio.br; ORCID https://orcid.org/0000-0001-9358-2115

${ }_{* * * * * * *}$ Doutor, Universidade Estácio de Sá, Cabo Frio, Rio de Janeiro, Brasil. Correio eletrônico: sergio.pinto@estacio.br; ORCID https://orcid.org/0000-0002-5588-9260

${ }_{* * * * * * * *}$ Doutor, Universidade do Estado do Rio de Janeiro, Rio de Janeiro, Brasil. Correio eletrônico: rodolfoalkmim@gmail.com; ORCID https://orcid.org/0000-0001-9707-2649

********* Doutor, Universidade Estácio de Sá, Cabo Frio, Rio de Janeiro, Brasil. Correio eletrônico: carlos.pernambuco@estacio.br; ORCID https://orcid.org/0000-0003-2915-6669

${ }^{* * * * * * * * * *}$ Doutor, Universidade Estácio de Sá, Cabo Frio, Rio de Janeiro, Brasil. Correio eletrônico: rodrigo.vale@estacio.br; ORCID https://orcid.org/0000-0002-3049-8773
}

Revista de Investigación Cuerpo, Cultura y Movimiento

ISSN: 2248-4418 | e-ISSN: 2422-474X | DOI: https://doi.org/10.15332/2422474X

Vol. 11 N.o 2 | julio-diciembre de 2021 
Citar como:

Rodrigues, D. B. A., Seixas-da-Silva, I. A., Brum, R. S. O., Dublasievicz, R. M., Principe, V. A., Pinto, S. M., Nunes, R. A. M., Pernambuco, C. S. y Vale, R. G. S. (2021). Desenvolvimento motor em escolares praticantes e não praticantes de dança. Revista de Investigación Cuerpo, Cultura y Movimiento, 11(2). https://doi.org/10.15332/2422474X.6876

\section{(C) $(1)(9)$}

\section{Resumo}

O estudo teve como objetivo comparar o equilíbrio e a motricidade global de escolares praticantes de Educação Física curricular e praticantes de balé como atividade física extracurricular. $\mathrm{O}$ estudo foi descritivo comparativo. Foi aplicada a Escala de Desenvolvimento Motor, especificamente os testes de equilíbrio e motricidade global, em 18 crianças na faixa etária de 9 a 11 anos, das quais 9 eram praticantes de balé e 9, não praticantes de atividade física extracurricular. Para a comparação do desenvolvimento motor entre os grupos de estudo, aplicou-se o teste de Fischer. O nível de p<0,05 foi considerado para a significância estatística. Os resultados da pesquisa mostraram que não houve diferenças significativas entre os grupos balé e escola nos testes de equilíbrio e motricidade global. Com esta pesquisa, conclui-se que os indivíduos analisados no estudo apresentaram o mesmo nível de desenvolvimento motor em virtude de todos os grupos atingirem mais de $50 \%$ de itens positivos.

Palavras-chave: desempenho psicomotor, dança, instituições acadêmicas. 


\section{Desempeño motriz en escolares practicantes y no practicantes de danza}

\section{Resumen}

El propósito del estudio fue comparar el equilibrio y la motricidad global de escolares practicantes de Educación Física curricular y practicantes de balet como actividad física extracurricular. El estudio fue descriptivo comparativo. Se aplicó la Escala de Desarrollo Motor, específicamente las pruebas de equilibrio y motricidad global, a 18 niños entre 9 y 11 años, de los que nueve eran practicantes de balet y nueve, no practicantes de actividad física extracurricular. Para comparar el desarrollo motor entre los grupos de estudio, se aplicó la prueba de Fischer. Para la significancia estadística se consideró el nivel de p<0,05. Los resultados de la investigación evidenciaron que no hubo diferencias significativas entre los grupos balet y escuela en las pruebas de equilibrio y motricidad global. Con la investigación, se concluye que los individuos analizados en el estudio presentaron el mismo nivel de desarrollo motor debido a que todos los grupos alcanzaron más de $50 \%$ de ítems positivos.

Palabras clave: desempeño psicomotor, danza, instituciones académicas.

\section{Motor performance in schoolchildren practicing and not practicing dance}

\section{Abstract}

The purpose of the study was to compare the balance and global motor skills of schoolchildren practicing curricular Physical Education and ballet as an extracurricular physical activity. The study was descriptive comparative. The Motor Development Scale, specifically the balance and global motor skills tests, was applied to 18 children between 9 and 11 years of age, nine of whom practiced ballet and nine who did not practice extracurricular activities. To compare motor development between the study groups, Fischer's test was applied. For statistical significance, the 
$\mathrm{p}<0.05$ level was considered. The research results showed that there were no significant differences between the ballet and school groups in the balance and global motor skills tests. With the research, it is concluded that the individuals analyzed in the study presented the same level of motor development due to the fact that all groups reached more than $50 \%$ of positive items.

Keywords: psychomotor performance, dance, schools.

\section{Introdução}

De acordo com o Instituto Nacional de Estudos e Pesquisas Educacionais Anísio Teixeira, no Censo Escolar da Educação Básica (2018), o número de alunos matriculados no ensino fundamental em 2018 foi de 27.183.970, dos quais $55,83 \%$ correspondem ao número de alunos matriculados nos anos iniciais do referido ensino.

Segundo os Parâmetros Curriculares Nacionais (PCN), o aluno do ensino fundamental deve estar ciente de que os benefícios para a sua saúde emanam da prática de atividade física regular (PCN, 1997). Nesse sentido, a Educação Física escolar possibilita, de acordo com a Base Nacional Curricular Comum (BNCC), uma série de conhecimentos culturais para os alunos, com experiências lúdicas, emotivas, saberes corporais e estéticos. Além de oferecer práticas lúdicas em sua vivência escolar, instiga os alunos a participarem de forma independente das atividades de lazer e saúde (BNCC, 2018). Cada prática corporal oferece à criança um tipo de conhecimento que ela só tem acesso nessa disciplina. Esse conhecimento é tão peculiar que se torna insubstituível através dos processos de problematização, desnaturalização e evidencialização da multiplicidade de sentido. Isso faz com que o aluno seja capaz de transmitir todo o seu conhecimento através de suas práticas corporais (BNCC, 2018). 
Tais práticas necessitam de um espaço escolar planejado, visto que este pode proporcionar um ambiente de rendimentos ou de barreiras. Tanto o ato de aprender quanto o de ensinar exigem condições favoráveis ao bemestar do professor e do aluno, pois é por meio dele que se propaga a prática pedagógica (Ribeiro, 2004).

Um ambiente diversificado proporciona para a criança um bom desenvolvimento motor, pois diferentes estímulos fazem com que a relação com ele ocorra de diversas maneiras, possibilitando novas situações. Quanto maior a quantidade de estímulos, melhor será a aprendizagem motora, porque essa aprendizagem será potencializada, visto que serão estimuladas diferentes áreas do cérebro, e a comunicação entre essas áreas faz com que a criança tenha um melhor desempenho na aprendizagem de tarefas complexas (Silva et ál., 2016).

O desenvolvimento motor ocorre de forma progressiva mediante processos contínuos e dinâmicos ao longo da vida. Assim, a obtenção de padrões motores, com o desenvolvimento da composição espaço-temporal dos segmentos corporais, promove transformações na forma de realizar tarefas (Campelo, 2013).

Explicar como os diversos componentes musculoesqueléticos são coordenados a fim de garantir uma ação efetiva é um desafio para os pesquisadores do comportamento motor. O ponto básico a respeito da coordenação motora se refere a como uma pessoa relaciona as partes do corpo, uma com outra, durante a execução de uma tarefa a partir de informações adquiridas do meio. É de extrema importância a atividade motora no desenvolvimento global de um indivíduo, principalmente no início da vida, pois a maturação do sistema nervoso central ocorre nessa fase. Quando há ausência de estímulos corporais e ambientais, algumas crianças tendem a apresentar dificuldades em outras fases do seu desenvolvimento (Amorim et ál., 2006; Latini et ál., 2006). 
As relações entre ambiente e aprendizagem estão associadas com as alterações nos processos cognitivos, assim como com a maturação e a plasticidade do sistema nervoso central. A importância da neuropsicologia está cada vez mais fortalecida, visto que é uma área de estudo preocupada com a formação tanto de medidas do desenvolvimento como de indicadores de dificuldades nesse processo. Medidas essas que são fundamentais para possibilitar a inclusão de meios que possam intervir na estimulação de habilidades ao decorrer do desenvolvimento (Carreiro et ál., 2015).

O conhecimento da neurociência pode auxiliar nos desafios de aprendizagem e oferecer estímulos unidos aos fatores característicos dos alunos, o que garantiria um desenvolvimento adequado de suas habilidades e competências (Simões Neto et ál., 2018).

De acordo com Betti e Zuliani (2002), a Educação Física veio para integrar-se à educação intelectual e moral. Cada vez mais, o esporte tem sido praticado por meio de danças, da ginástica artística, dos esportes de luta, fazendo com que haja maiores informações divulgadas sobre o assunto. Porém, é grande o número de crianças que preferem assistir a um esporte a praticá-lo, o que faz com que haja uma diminuição no seu desempenho motor.

É essencial, para o desenvolvimento da criança, que haja estímulo para a realização de atividades motoras. As habilidades motoras de uma criança devem evoluir conforme seu crescimento maturacional, tornando-se cada vez mais completas e complexas (Rosa Neto, 2002).

A Educação Física escolar deve preparar o aluno para integrá-lo à cultura corporal do movimento, fazendo com que ele saiba os benefícios do exercício à sua saúde e seja capaz de se posicionar perante as novas formas de praticar atividade física (Betti e Zuliani, 2002). 
Nesse contexto, considera-se atividade física todo movimento corporal produzido pela musculatura esquelética, o qual ocasiona gasto de energia. Tais atividades praticadas de forma adequada, com objetivos definidos e bem planejadas oferecem diversos benefícios à saúde, modificando os componentes estruturais e fisiológicos do corpo humano (Barbosa, 2017).

Entre a variedade de atividades físicas, a dança oferece para os seus praticantes benefícios, como fortalecimento dos músculos e prevenção de problemas posturais, além de trabalhar o desenvolvimento motor nas crianças (Tiecher et ál., 2015) em seus principais elementos: motricidade global, motricidade fina, equilíbrio, lateralidade e esquema corporal (Maciel et ál., 2015).

Entende-se motricidade global quando diversas funções motoras se interagem, por exemplo, a coordenação e a lateralidade, a interação entre a tonicidade e o equilíbrio, entre outros. É importante respeitar o ritmo individual de cada criança para que os seus gestos motores sejam sempre de qualidade, pois cada uma tem seu próprio tempo de desenvolvimento (Rosa Neto, 2002).

Para Rosa Neto (2002), todas as ações dos segmentos corporais são baseadas no equilíbrio. Uma criança pequena, quando recebe estímulos, adota uma postura e reage por reflexo; a existência do equilíbrio se dá após a pessoa conseguir manter posições, posturas e atitudes.

A dança, além de auxiliar no desenvolvimento das habilidades motoras das crianças, pode proporcioná-las não só uma evolução pedagógica, mas também uma evolução psicológica, pois as leva a aprender de forma livre por meio de suas experiências corporais, culturais e artísticas. Assim, contribui para a formação individual e coletiva de relacionar-se com o outro, visto que a criança aprende a respeitar o tempo e o espaço de cada um (Santos, 2011). 
O ensino da dança deve ir além de cópias de passos, despertando, no aluno, o interesse de criar, executar, compreender e contextualizar o movimento (Vieira, 2014). Esta é capaz de promover a qualidade de vida, o aperfeiçoamento das qualidades físicas, a consciência corporal e tem um papel significativo no desenvolvimento físico-motor. Sabe-se ainda que a comunicação não verbal da dança, que compõe o universo da cultura corporal do movimento, reflete os sentimentos e as emoções dos dançarinos, fazendo com que a dança seja uma prática pedagógica que contribuiu, positivamente, como metodologia de ensino com objetivo educacional (Lima et ál., 2012).

Em meio à dança, o balé ganha destaque por proporcionar a autoestima, a autoconfiança, a agilidade mental e o equilíbrio entre o corpo e a mente (Tiecher et ál., 2015). Rodrigues et ál. (2018) afirmam que a prática do balé tem uma importância significativa para o desenvolvimento cognitivo, afetivo, social e motor de uma criança.

Este trabalho se justifica em função de possibilitar níveis observacionais que venham a contribuir com o desenvolvimento motor de escolares da faixa etária de 9 a 11 anos que participam da Educação Física curricular associada à atividade extracurricular do balé. Este trabalho também pode servir de apoio científico para os profissionais da área de Educação Física que atuam nas escolas e em centros de atividades físicas, por apresentar informações que venham contribuir para o aperfeiçoamento profissional. Assim, o presente trabalho tem por objetivo comparar o equilíbrio e a motricidade global entre escolares que praticam Educação Física curricular e o balé. 


\section{Metodologia}

A pesquisa correlacional é um tipo de pesquisa que explora as possíveis relações entre as variáveis, exceto a relação de causa-efeito, e o estudo descritivo estabelece a ligação entre as variáveis e seu desempenho (Thomas et ál., 2012).

A população do estudo se caracterizou por alunas devidamente matriculadas em escolas públicas e particulares que praticam a Educação Física escolar e o balé como atividade física extracurricular.

Para os critérios de inclusão, definiram-se alunas praticantes de balé e da Educação Física escolar e alunas que praticam a Educação Física escolar, e não fazem nenhuma atividade física extracurricular, na faixa etária de 9 a 11 anos. O estudo adotou os seguintes critérios de exclusão: alunos do gênero masculino; meninas praticantes de alguma atividade física extracurricular; pessoas com necessidades especiais.

Após o processo de amostragem, a amostra então foi constituída por nove alunas praticantes de balé e nove alunas não praticantes de atividade física extracurricular.

A pesquisa foi desenvolvida conforme as recomendações da Resolução 580/2018 do Conselho Nacional de Saúde sobre pesquisa com seres humanos. Todos os participantes foram avisados e tomaram consciência do que seria feito, e concordaram em participar. Os responsáveis das crianças autorizaram a coleta de dados.

Para a coleta de dados, foi utilizado o teste Escala de Desenvolvimento Motor (EDM) de Francisco Rosa Neto (2002), especificamente os testes de equilíbrio e motricidade global. Os testes foram aplicados de acordo com a idade de cada criança. Além da EDM, também foram coletados dados de idade (anos), estatura (metros), massa corporal (kg) e índice de massa 
corporal (IMC, em kg/m²), com o objetivo de caracterizar a amostra do estudo.

Para crianças de 9 anos, o teste de equilíbrio consiste em manter-se sobre o pé esquerdo, a planta do pé direito apoiada sobre o joelho esquerdo, as mãos fixadas nas coxas e os olhos abertos. No teste de motricidade global, as crianças devem saltar no ar, flexionar os joelhos e tocar os calcanhares com as mãos.

No teste de equilíbrio, as crianças de 10 anos devem manter-se sobre a ponta dos pés, os olhos fechados, os braços ao longo do corpo, os pés e as pernas juntas. E, no de motricidade global, devem flexionar o joelho em ângulo reto e manter os braços relaxados ao longo do corpo. A $25 \mathrm{~cm}$ do pé que repousa no solo, coloca-se uma caixa de fósforos. A criança deve levála impulsionando-a com o pé até o ponto situado a $5 \mathrm{~m}$.

No teste de equilíbrio, as crianças de 11 anos deviam ficar com os olhos fechados, manter-se sobre a perna direita, o joelho esquerdo flexionado em ângulo reto, a coxa esquerda paralela à direita e em ligeira abdução, os braços ao longo do corpo e, após 30 segundos de descanso, realizar o mesmo exercício com a outra perna. Para o de motricidade global, deveriam saltar sobre uma cadeira de $45 \mathrm{~cm}$ a $50 \mathrm{~cm}$, com uma distância de $50 \mathrm{~cm}$ dela. $\mathrm{O}$ encosto foi sustentado pelo examinador.

Para a análise dos resultados, foi utilizado o programa IBM SPSS Statistics 23 for Windows. Os dados foram apresentados como média, desvio-padrão e frequência absoluta e relativa. Aplicou-se o teste de Fischer para a comparação do desenvolvimento motor entre os grupos de estudo. O nível de $\mathrm{p}<0,05$ foi considerado para a significância estatística. 


\section{Resultados e discussão}

A tabela 1 apresenta as características da amostra referentes às variáveis idade, massa corporal, estatura e IMC.

Tabela 1. Características da amostra $(n=18)$

\begin{tabular}{|c|c|c|c|}
\hline & Balé $(n=9)$ & Escola $(n=9)$ & Geral $(n=18)$ \\
\hline & Média \pm DP & Média \pm DP & Média \pm DP \\
\hline Idade (anos) & $9,78 \pm 0,83$ & $9,78 \pm 0,83$ & $9,77 \pm 0,80$ \\
\hline Massa corporal (kg) & $44,7 \pm 9,71$ & $50,24 \pm 11,16$ & $47,47 \pm 10,54$ \\
\hline Estatura (m) & $1,45 \pm 0,10$ & $1,49 \pm 0,04$ & $1,46 \pm 0,07$ \\
\hline IMC $\left(\mathrm{kg} / \mathrm{m}^{2}\right)$ & $21,18 \pm 2,89$ & $22,52 \pm 4,12$ & $21,85 \pm 3,51$ \\
\hline
\end{tabular}

DP: desvio-padrão; n: número da amostra.

Fonte: elaboração própria

A tabela 2 mostra que não houve diferença significativa tanto no teste de equilíbrio quanto no de motricidade global, porém nota-se que o grupo balé obteve maior frequência de acertos em ambos os testes.

Tabela 2. Análise comparativa dos resultados dos testes de equilíbrio e motricidade global nos grupos balé e escola

\begin{tabular}{|c|c|c|c|c|c|}
\hline & \multicolumn{2}{|c|}{ Balé } & \multicolumn{2}{|c|}{ Escola } & \multirow{2}{*}{$\begin{array}{c}\text { Teste de } \\
\text { Fisher } \\
\text { Valor-p }\end{array}$} \\
\hline & $\begin{array}{l}\text { Freq. } \\
\text { absoluta }\end{array}$ & $\begin{array}{l}\text { Freq. } \\
\text { relativa }\end{array}$ & $\begin{array}{l}\text { Freq. } \\
\text { absoluta }\end{array}$ & $\begin{array}{l}\text { Freq. } \\
\text { relativa }\end{array}$ & \\
\hline Equilíbrio & 8 & $88,80 \%$ & 7 & $77,77 \%$ & 0,999 \\
\hline $\begin{array}{c}\text { Motricidade } \\
\text { global }\end{array}$ & 8 & $88,80 \%$ & 5 & $55,55 \%$ & 0,444 \\
\hline
\end{tabular}

Freq: frequência.

Fonte: elaboração própria.

O presente estudo não encontrou diferenças significativas entre os grupos balé e escola nos testes de equilíbrio e motricidade global. O mesmo resultado foi encontrado no estudo de Arruda e Silva (2009) com 21 escolares na faixa etária de 4 a 6 anos. Após uma aplicação de atividades 
lúdicas para contribuir para o desenvolvimento psicomotor das crianças, os autores não encontraram diferenças significativas entre o teste e o reteste nas valências de equilíbrio e motricidade global. No presente estudo, o mesmo resultado foi encontrado, talvez por não ter havido tempo para que fosse aplicado o teste e o reteste, portanto não sendo possível avaliar modificações mensuráveis na motricidade.

Comparando também com o estudo de Costa e Silva (2009), pode-se notar que houve uma semelhança nos resultados dos testes. A aplicação da EDM (2002) ocorreu com 14 escolares de 4 a 9 anos de idade, para os quais a aula de Educação Física escolar apenas não foi suficiente para que os alunos pudessem ter uma diferença relevante nos quocientes motores das habilidades de equilíbrio e motricidade global no período de 30 meses entre a $1^{\mathrm{a}}$ e a $2^{\mathrm{a}}$ avaliação. Embora para o presente estudo não tenham sido feitos teste e reteste, a provável justificativa para que esse resultado tenha sido encontrado pode estar pela participação frequente e estimulação motora diversificada durante as aulas de Educação Física escolar.

Oliveira (2013) realizou um estudo para avaliar o desempenho das habilidades motoras motricidade global, motricidade fina e lateralidade, de escolares do $3^{\circ}$ e $4^{\circ}$ ano do ensino. Os alunos do $3^{\circ}$ ano foram submetidos às aulas de Educação Física planejadas e estruturadas, enquanto os do $4^{\circ}$ ano tinham aula de recreação livre. Notou-se então que os alunos do $3^{\circ}$ ano tiveram resultados melhores nos testes. Tais resultados apontam que, quando as aulas são bem estruturadas e planejadas, há uma potencialização no desenvolvimento motor. Essa potencialização pode ter ocorrido também nas aulas de Educação Física escolar do presente estudo, visto que o nível de desenvolvimento motor encontrado foi semelhante ao grupo que praticava uma atividade física extracurricular. 
Em um estudo feito com crianças com necessidades educativas especiais, usando a aula de balé uma vez por semana com 90 minutos de aula para a verificação de mudança no comportamento motor, detectaram-se diferenças estatisticamente significativas $(\mathrm{p}<0,05)$ entre pré e pós-testes nas variáveis motricidade global, equilíbrio e outras (De Oliveira et ál., 2002). Visto que o grupo amostral era diferente do do presente estudo, para eles, uma estimulação diferenciada apresentava uma mudança comportamental em um curto espaço de tempo. Isso pode justificar o motivo pelo qual esse resultado se contrapõe ao do presente estudo. Utilizando a aula de dança como meio de intervenção, Cardoso et ál. (2016) tiveram como amostra um grupo de 17 crianças (nove meninas e oito meninos) de uma escola municipal do estado de Roraima, Brasil, que, em um período de 24 semanas, fizeram aula de dança duas vezes por semana. Os autores aplicaram os testes da bateria Rosa Neto para avaliar a coordenação motora, o equilíbrio, o esquema corporal e a organização espacial e temporal antes do início e após o período de intervenção, e tiveram como resultado, na variável equilíbrio, uma evolução significativa nos escores na idade motora de 48,5\% nos meninos (7,25 \pm 1,99 para 9,62 $\pm 1,07)$ e $45 \%$ nas meninas $(7,00 \pm 2,07$ para 9,22 $\pm 0,67)$. Na coordenação motora global, apenas os meninos apresentaram evolução significativa de $24,6 \%(8,38 \pm 1,07$ para 9,12 $\pm 0,36)$, nas meninas foi de $23,4 \%(7,78 \pm$ 1,64 para 9,11 $\pm 0,79$ ). Essa contradição ao presente estudo pode ser justificada pelo fato de o estudo de Cardoso Silva e Galiasso (2016) ter acompanhado a amostra por semanas, enquanto no presente, o teste foi feito apenas no início do $2^{\circ}$ trimestre do ano.

Santos et ál. (2015), ao avaliarem crianças de 7 a 10 anos de idade praticantes de balé, obtiveram os resultados de motricidade global e equilíbrio em escores superiores ( $>50 \%$ ) quando comparadas com as crianças pertencentes exclusivamente à Educação Física escolar. Tais 
resultados contradizem com o presente estudo, pois, embora não façam uma atividade física sistematizada, as crianças avaliadas do grupo escola apresentam bastante vivência e estimulação motora, uma vez que os dois grupos avaliados apresentam escores maiores que $50 \%$.

Porém, assim como no presente estudo, Espírito Santo et ál. (2016) investigaram 68 crianças entre 4 e 5 anos de idade para avaliar a correlação das variáveis dança e desempenho motor entre um grupo que pratica dança curricular no ambiente escolar e outro grupo que não pratica dança. Os autores encontraram como resultado que a dança não interfere no desempenho motor global da criança. Esse resultado corrobora o presente estudo. Isso pode ser justificado talvez pelo número reduzido da amostra e pelo meio em que a criança vive poder interferir em seu desenvolvimento motor.

O equilíbrio é uma das capacidades físicas que se desenvolve na infância que é essencial para qualquer movimento (Silveira et ál., 2006). Turcatto et ál. (2013), em uma pesquisa com um grupo de escolares de 6 a 8 anos de idade de uma escola particular do município de Venâncio Aires, Rio Grande do Sul, Brasil, dividiram os participantes em grupo experimental (GE) e grupo controle (GC), com 24 alunos em cada grupo. Em ambos os grupos, foram aplicados pré e pós-teste motores e de aptidão física, e ao grupo experimental aplicadas 21 sessões de intervenção por meio da dança. O GE, quando comparado ao GC, teve maiores ganhos referente ao equilíbrio. Resultado esse que contrapõe o presente estudo. Isso pode ser justificado pela idade da amostra e porque, neste estudo, as intervenções não foram controladas.

Silva (2016), em uma pesquisa com 10 escolares de 6 e 7 anos de idade da cidade de Paulo Afonso, Bahia, Brasil, dos quais cinco eram do gênero feminino e cinco, do masculino, realizou o teste de Rosa Neto (2002) no início do $1^{\circ}$ bimestre e, durante as aulas de Educação Física desse mesmo 
bimestre, fez o uso da dança para avaliar se haveria melhora no desempenho motor dos estudantes ao final do período. Como resultado, obteve uma melhora significativa apenas no teste de equilíbrio, em que, no início do bimestre, apenas dois alunos conseguiram realizar o teste e, no final do bimestre, todos os 10 alunos lograram sucesso. Thiesen e Sumiya (2011) destacam que a dança proporciona um desempenho somatossensorial que tem como resultado uma melhora na estabilidade corporal. Esses resultados contradizem o presente estudo, o que pode ser justificado pelo gênero da amostra e por ter ocorrido um período de intervenção.

\section{Conclusões}

Considerando os resultados obtidos neste estudo, conclui-se que os indivíduos analisados apresentaram o mesmo nível de desenvolvimento motor em virtude de todos os grupos atingirem mais do que 50\% de itens positivos. Isso indica que a Educação Física escolar e a educação física associada ao balé atendem ao mesmo nível de desenvolvimento motor na amostra estudada.

Deve-se levar em consideração que o número da amostra foi pequeno, que não foi feito um acompanhamento antes da coleta dos dados e que, embora a criança não faça atividade física extracurricular sistematizada, ela pode ter uma diversidade de estímulos e vivências motoras que potencializam o desenvolvimento motor, o que pode justificar os resultados encontrados no presente estudo.

Sugere-se que futuros estudos desenvolvam investigações com um tamanho amostral maior, controlando a intervenção e com a utilização comparativa com outras modalidades de atividade física. 


\section{Referências}

Amorim, L. A. C., Zogaib, F. G., Seixas da Silva, I. A., Brüch, V. L. e Dantas, P. M. S. (2006). Estudo descritivo do comportamento psicomotor de indivíduos de ambos os gêneros participantes de um projeto esportivo. Coleção Pesquisa em Educação Física (v. 4, pp. 422-427). Editora Fontoura.

Arruda, K. M. F. e Silva, E. A. A. (2009). Desenvolvimento motor na educação infantil através da ludicidade. Connection line, 4, 37-50. https://doi.org/10.18312/19807341.n4.2009.131

Barbosa, R. C. (2017). Estudo comparativo de força e flexibilidade em praticantes e não praticantes do balé clássico em Campina Grande-PB. (Trabalho de conclusão de curso em educação física). Universidade Estadual da Paraíba, Paraíba, Brasil. https://doi.org/10.14393/19834071.v26.n2.2017.35629

Brasil (1997). Parâmetros Curriculares Nacionais: Educação Física. Secretaria de Educação Fundamental. Ministério da Educação e Cultura.

http://portal.mec.gov.br/seb/arquivos/pdf/fisica.pdf

Brasil (2018). Base Nacional Curricular Comum. Ministério da Educação do Brasil. http://basenacionalcomum.mec.gov.br/images/BNCC EI EF 110518 versaofinal site.pdf

Betti, M. e Zuliani, R. (2002). Educação física escolar: uma proposta de diretrizes pedagógicas. Revista Mackenzie de educação física e esporte, 1(1), 73-81. https://doi.org/10.1590/s1807-55092011000500011

Campelo, A. M. (2013). A realidade virtual na intervenção motora em crianças com transtorno do desenvolvimento da coordenação. (Dissertação de mestrado em desenvolvimento humano e tecnologias). Universidade Estadual Paulista Júlio de Mesquita Filho, São Paulo, Brasil. https://doi.org/10.11606/t.39.2013.tde28112013-151731

Cardoso, J. B., Silva, R. G. e Galiasso, C. A. F. (2016). Contribuições da dança no desenvolvimento motor de escolares. Anais XI Semana Nacional de Ciência e Tecnologia no Estado de Roraima, Roraima, Brasil.

https://doi.org/10.29327/15284

Revista de Investigación Cuerpo, Cultura y Movimiento ISSN: 2248-4418 | e-ISSN: 2422-474X | DOI: https://doi.org/10.15332/2422474X 
Carreiro, L. R. R., Reppold, C. T., Mariani, M. M. de C., Lellis V. R. R., Dias, N. M., Fioravante-Bastos, A. C. M., Seabra, A. G. (2015). Habilidades cognitivas ao longo do desenvolvimento: contribuições para o estudo da atenção concentrada. Psicologia: teoria e prática, 17(2), 153-170. https://doi.org/10.15348/19806906/psicologia.v17n2p153-170

Costa, R. M. e Silva, E. A. A. (2009). Escala de desenvolvimento motor de Rosa Neto: estudo longitudinal em uma escola da rede particular de ensino de Cuiabá-MT. Connection Line, 4, 51-64. https://doi.org/10.18312/1980-7341.n4.2009.133

Instituto Nacional de Estudos e Pesquisas (2018). Censo Escolar da Educação Básica. Brasília: Ministério da Educação. http://portal.inep.gov.br/informacao-dapublicacao/-/asset publisher/6JYIsGMAMkW1/document/id/1487619

Latini, D. M., Seixas da Silva, I. A., Medeiros, H. B. O. e Firmino, M. M. (2006). Estudo descritivo e análise correlacional entre fatores psicomotores em jovens universitários da cidade de Niterói no estado do Rio de Janeiro. Coleção Pesquisa em Educação Física (v. 4, pp. 401-404). Editora Fontoura.

Lima, M. S., Fonseca, L. B., Azevedo, D. S., Ribeiro, R. S. L. (2012). A contribuição da dança para o desenvolvimento psicomotor na Educação Infantil. Revista digital efdesportes, 17(1875). https://www.efdeportes.com/efd175/danca-para-odesenvolvimento-psicomotor.htm

Maciel, C. L. A., Santo, L. P. E., Fernandes, C. T., Maciel, C. M. L. A. e Reis Filho, A. D. (2015). As contribuições da dança no desempenho motor de crianças da educação infantil. Arquivos em movimento, 11(o2), 29-46. https://revistas.ufrj.br/index.php/am/article/view/9257/pdf 59

Oliveira, L., Zancan, R. F., Krug, M. R. e Batistella, P. A. (2002). Dança e o desenvolvimento motor de portadores de necessidades educativas especiaisPNEEs. Revista educação especial, 20, 89-96. https://periodicos.ufsm.br/educacaoespecial/article/view/5122

Oliveira, G. M. (2013). O desenvolvimento motor nas séries iniciais do ensino fundamental de uma escola de Planaltina-DF. (Monografia de conclusão de curso em educação física). Universidade Federal de Goiás, Goiás, Brasil. https://doi.org/10.14393/19834071.2016.33168

Ribeiro, S. L. (2004). Espaço escolar: um elemento (in)visível no currículo. Sitientibus, 31, 103-118. http://www2.uefs.br:8081/sitientibus/pdf/31/espaco escolar.pdf

Revista de Investigación Cuerpo, Cultura y Movimiento ISSN: 2248-4418 | e-ISSN: 2422-474X | DOI: https://doi.org/10.15332/2422474X Vol. 11 N. 02 | julio-diciembre de 2021 
Rodrigues, M. F., Santos, L. F., Raymundo, L. X., Rocha, T. D. e Miguel, H. (2018).

Benefícios da prática do balé clássico para crianças de 08 a 14 anos da cidade de Mogi Guaçu. Revista científica multidisciplinar núcleo do conhecimento, 11(2), 6772.

https://doi.org/10.32749/nucleodoconhecimento.com.br/administracao/implanta cao-do-planejamento

Rosa Neto, F. (2002). Manual de avaliação motora. Artmed.

Santos, C. R., Silva, C. C., Damasceno, M. L., Medina-Papst, J. e Marques, I. (2015). Efeito da atividade esportiva sistematizada sobre o desenvolvimento motor de crianças de sete a 10 anos. Revista Brasileira de Educação Física e Esporte, 29(3), 497-506.

https://doi.org/10.1590/1807-55092015000300497

Santos, D. O. G. L. de O. (2011). O corpo que dança: a arte de brincar. (projeto de pesquisa em licenciatura em dança). Universidade Federal de Sergipe, Sergipe, Brasil. https://ri.ufs.br/handle/riufs/6778?locale=es

Silva, N. R. S. O. (2016). Dança e educação física: vivência e desenvolvimento motor no ensino fundamental I. Revista científica da Fasete, 11(2), 154-170.

https://doi.org/10.30612/hre.vgi16.13127

Silveira, C. R. A., Menuchi, M. R. P., Simões, C. S., Caetano, M. J. D. e Gobbi, L. T. B. (2006). Validade de construção em testes de equilíbrio: ordenação cronológica na apresentação das tarefas. Revista Brasileira de Cineantropometria e Desempenho Humano, 8(3), 66-72.

https://periodicos.ufsc.br/index.php/rbcdh/article/view/3911/3317

Simões Neto, J. C., Oliveira, G. F., Rocha, A. M. e Lopes, R. E. M. (2018). Associação entre o nível de atividade física e o desempenho cognitivo em crianças. Revista Multidisciplinar e de Psicologia, 12(39), 713-735. https://doi.org/10.14295/idonline.v12i39.1023

Tiecher, T. C., Toigo, A. M. e Rodrigues, L. G. C. (2015). Comparação das habilidades motoras fundamentais de locomoção de crianças entre 6 e 8 anos praticantes e não praticantes de balé. Cippus, 4(1), 36-54. https://doi.org/10.11606/d.5.2009.tdeo9122009-164929

Thiesen, T., Sumiya, A. (2011). Equilibrio e arco plantar no balé clássico. ConScientiae Saúde, 1O(1), 138-142.

https://periodicos.uninove.br/saude/article/view/2550/1906

Revista de Investigación Cuerpo, Cultura y Movimiento ISSN: 2248-4418 | e-ISSN: 2422-474X | DOI: https://doi.org/10.15332/2422474X Vol. 11 N.0 2 | julio-diciembre de 2021 
Thomas, J. R., Nelson, J. K. e Silverman, S. J. (2012). Métodos de pesquisa em atividade física. Artmed.

Turcatto, E. S., Burgos, M. Z., Reuter, C. P., Reckziegel, M. B. e Rosa, R. Q. (2013). Efeitos da dança no desenvolvimento motor e aptidão física: um estudo de caso em escolares. Revista digital efdesportes, 18(185).

https://www.efdeportes.com/efd185/efeitos-da-danca-e-aptidao-

fisica.htm\#: :text=Resultados\%20estes\%20que\%20corroboram\%200s\%20encont rados\%20nesta\%20pesquisa.\&text=Os\%20resultados\%20deste\%20estudo\%20apo ntam,de\%20forma\%20espec\%C3\%ADfica\%20na\%20flexibilidade

Vieira, M. S. (2014). A dança na arte e na educação física: diálogos possíveis. Revista Tempos e Espaços em Educação, 13, 177-185.

https://doi.org/10.20952/revtee.voio.3266 